\title{
Clinical Imaging Problem Identified
}

National Cancer Institute

\section{Source}

National Cancer Institute. Clinical Imaging Problem Identified. NCI Thesaurus. Code C92102.

Problems that occur with devices used for radiog raphic or imaging procedures e.g. CT scanners, magnetic resonance imaging. 